\title{
Der Staat als Glücksmaximierer?
}

\author{
von Bruno S. Frey, Alois Stutzer und Susanne Neckermann
}

Die ökonomische Analyse des Glücks ist ein neuer und vielversprechender Ansatz innerhalb der Ökonomie. Dieser Aufsatz gibt einen knappen Überblick über den Stand der ökonomischen Glücksforschung. Einige Determinanten menschlichen Wohlbefindens werden vorgestellt sowie mögliche Politikimplikationen diskutiert. Der Ansatz erlaubt es, öffentliche Güter direkt zu bewerten. Beispielhaft werden die negativen Konsequenzen von Terrorismus abgeschätzt. Darüber hinaus wird die Frage diskutiert, inwieweit die Maximierung des Glücksempfindens der Einwohner ein explizites Politikziel sein sollte.

The economics of happiness is a new and promising research avenue in economics. This paper presents a brief overview of the research in this area. We present selective evidence on the determinants of happiness and discuss some policy implications of the findings. The new approach allows the direct valuation of public goods, for example in the field of terrorism. Moreover, the question whether states should explicitly aim at maximising the happiness of their citizens is discussed.

\section{Glücksforschung heute}

Was macht ein gutes Leben aus? Was ist Glück? Diese Fragen beschäftigen die Menschheit seit Jahrtausenden. Einige Menschen betrachten das Glück als wichtigstes Ziel ihres Lebens. Für andere ist Glück einer unter mehreren wichtigen Aspekten eines guten Lebens. Menschliche Entwicklung (die auch Tugendhaftigkeit umfasst), Gerechtigkeit, Kameradschaft oder Freiheit stehen für diese Menschen auf gleicher Stufe wie das Glück als Ziel des Lebens.

Glück ist ein schwer fassbares, komplexes Konzept. Es ist daher schwierig und vielleicht sogar unmöglich, nach einer allgemeinen Definition von Glück und einem gelungenen Leben zu suchen. Ein anderer Ansatz besteht darin, die subjektive Sicht jedes Menschen anzuerkennen und es jedem Einzelnen selbst zu überlassen, was für ihn ein gutes Leben ausmacht. Das Glück der Menschen lässt sich dennoch erfassen und analysieren: Man kann die Leute direkt fragen, wie zufrieden sie mit ihrem Leben sind. Damit wird unterstellt, dass die Individuen am besten beurteilen können, ob sie glücklich oder unglücklich sind, ob sie die Qualität ihres Lebens als befriedigend einschätzen oder nicht. Dieser Ansatz entspricht der grundlegenden Tradition in der Wirtschaftswissenschaft, das Indi- 
viduum als Handlungs- und zentrale Analyseeinheit zu betrachten. Die Befragung der Menschen nach ihrem subjektiven Wohlbefinden bildet die Grundlage für eine der neuesten Forschungsrichtungen in der Volkswirtschaftlehre, die ökonomische Analyse des Glücks. Die folgenden Ausführungen sollen den Zugang zur ökonomischen Forschung zum Thema Glück öffnen. ${ }^{1}$

Im zweiten Teil werden die Grundzüge des ökonomischen Ansatzes zur Analyse des subjektiven Wohlbefindens skizziert. Der dritte zeigt einige Fragen auf, die mit dem neuen Ansatz angegangen werden können. Im vierten Teil wird ein neues Instrument vorgestellt, um die individuelle Wohlfahrt im Kontext der Kosten-Nutzen-Analyse zu untersuchen. Der Lebenszufriedenheitsansatz ermöglicht es, die Auswirkungen öffentlicher Güter und Übel auf den Nutzen (bzw. Disnutzen) des Einzelnen direkt zu erfassen. Dies wird am Beispiel eines zentralen öffentlichen Übels, dem Terrorismus, illustriert. Im abschließenden fünften Teil wird die Frage diskutiert, ob der Staat das Glück seiner Bürger und Bürgerinnen maximieren soll.

\section{Glück aus ökonomischer Sicht}

Laut der traditionellen Wirtschaftstheorie braucht es kein kardinales und interpersonell vergleichbares Maß des Nutzens, um zu analysieren, wie Individuen auf Veränderungen relativer Preisen reagieren. Dem axiomatischen Ansatz der offenbarten Präferenzen (revealed preferences) folgend, liefern die getroffenen beobachtbaren Entscheidungen alle notwendigen Informationen, um auf den Nutzen der verschiedenen Handlungsalternativen zu schließen. Das Wohlfahrtsniveau einzelner Menschen braucht dafür nicht direkt berechnet zu werden. Diese Sichtweise herrscht in den Wirtschaftswissenschaften noch immer vor.

Zahlreiche Forscher haben diesen Ansatz jedoch aus verschiedenen Gründen in Frage gestellt. Tatsächlich gibt es in der Ökonomik viele Beispiele für nicht-

1 Weiterführende Darstellungen aus ökonomischer Perspektive mit Hinweisen auf eine Vielzahl von Forschungsarbeiten finden sich zum Beispiel bei Clark, A./Frijters, P./Shields, M.: Relative Income, Happiness, and Utility: An Explanation for the Easterlin Paradox and Other Puzzles, in: Journal of Economic Literature, 46/1 (2008), 95-144; Di Tella, R./MacCulloch, R. J.: Some Uses of Happiness Data in Economics, in: Journal of Economic Perspectives, 20/1 (2006), 25-46; Dolan, P./Peasgood, T./White, M.: Do We Really Know what Makes Us Happy? A Review of the Economic Literature on the Factors Associated with Subjective Well-Being, in: Journal of Economic Psychology, 29/1 (2008), 94-122; Frey, B.S.Stutzer, A.: Happiness and Economics: How the Economy and Institutions Affect WellBeing, Princeton, NJ, 2002; id.: What Can Economists Learn from Happiness Research?, in: Journal of Economic Literature, 40/2 (2002), 402-435; Frey, B. S.: Happiness: A Revolution in Economics, Cambridge, MA, 2008; Frey, B.S./Marti, C.: Glück: Die Sicht der Ökonomie, Zürich, 2010, i.E. 
objektivistische theoretische Analysen. Sie berücksichtigen Emotionen, Zielerfüllung, Sinngebung, Selbstbild und Status. In der umfangreichen Literatur zu Entscheidungsanomalien wird bezweifelt, ob sich der Nutzen generell aus den beobachteten Entscheidungen ableiten lässt. Die gleichen Probleme treten bei intertemporalen Entscheidungen auf, wenn Individuen ungenügende Selbstkontrolle haben. ${ }^{2}$ Das Vertrauen der ökonomischen Standardtheorie in einen objektivistischen Ansatz lässt sich somit weder theoretisch noch empirisch aufrechterhalten. Überdies schränkt der traditionelle Ansatz die Möglichkeit ein, menschliches Wohlbefinden zu verstehen und zu beeinflussen.

\section{Verschiedene Ansätze zum subjektiven Nutzen}

Die Bestimmung des subjektiven Nutzens ermöglicht, menschliches Wohlbefinden unmittelbar einzuschätzen. Abhängig vom gewählten empirischen Ansatz wird eine stärker hedonistische Interpretation des Nutzens oder eine gesamthafte Beurteilung der Zufriedenheit mit dem eigenen Leben gewählt. Der hedonistische Ansatz kommt insbesondere im Begriff des „erfahrenen Nutzens“ (experienced utility) zum Ausdruck. ${ }^{3}$

Auch der zweite Ansatz entspricht einer subjektiven Betrachtung des Nutzens. Diese erkennt an, dass jeder Mensch eigene Vorstellungen vom Glück und von einem guten Leben hat. Dieser Sichtweise folgend, lässt sich das individuelle Glück erfassen und analysieren, indem die Menschen gefragt werden, wie zufrieden sie mit ihrem Leben sind. Damit wird der Einschätzung der unmittelbar beteiligten Personen vertraut: Menschen sind fähig, die Gesamtqualität ihres Lebens zu beurteilen. Beide Ansätze eignen sich für zahlreiche Fragestellungen gut, weil sie das subjektive Wohlbefinden direkt messen, anstelle dieses allein aus den getroffenen Entscheidungen abzuleiten.

2 Kritik an zahlreichen Aspekten der traditionellen Ökonomie findet sich in der sogenannten ,Verhaltensökonomik“ oder „Ökonomie und Psychologie“ (vgl. etwa Camerer, C./Loewenstein, G./Rabin, M. (Hg.) Advances in Behavioral Economics, New York/Princeton, 2003; Frey, B. S./Stutzer, A.: Economics and Psychology: from Imperialism to Inspired Economics, in: Philosophie économique, 2/4 (2001), 5-22; id.: Economics and Psychology. A Promising New Cross-Disciplinary Field, Cambridge, MA, 2007; Rabin, M.: Psychology and Economics, in: Journal of Economic Literature, 36/1 (1998), 11-46.

3 Kahneman, D./Wakker, P.P./Sarin, R.: Back to Bentham? Explorations of Experienced Utility, in Quarterly Journal of Economics, 112/2 (1997), 375-405. 


\section{Quantifizierung des subjektiven Wohlbefindens}

In den letzten Jahren hat die Messung des subjektiven Wohlbefindens dank umfassender Arbeiten zahlreicher Psychologen große Fortschritte gemacht. ${ }^{4}$ Es wurden verschiedene Indikatoren subjektiven Wohlbefindens entwickelt, die auf unterschiedlichen Messtechniken beruhen:

- umfassende Selbsteinschätzungen der individuellen Lebenszufriedenheit, die auf repräsentativen Umfragen beruhen;

- die Erlebnis-Stichproben-Methode (Experience Sampling Method, ESM), die Informationen zur tatsächlichen Erfahrung von Individuen in Echtzeit in ihrer natürlichen Umgebung sammelt, und

- die Methode der Tagesrekonstruktion (Day Reconstruction Method), bei der Menschen gebeten werden, sich bewusst zu machen, wie zufrieden sie sich in verschiedenen Situationen im Laufe eines Tages fühlten. ${ }^{5}$

Ein Grossteil der bisherigen empirischen Glücksforschung basiert auf repräsentativen Erhebungen darüber, wie Individuen ihre Lebenszufriedenheit einschätzen. Eine typische Frage lautet: „Ganz allgemein gefragt - wie zufrieden sind Sie mit dem Leben, das Sie führen?" Die Antwortmöglichkeiten reichen auf einer Skala von 0 (,gar nicht zufrieden“) bis 10 (,,vollumfänglich zufrieden“). Dieser Erhebungsansatz hat ein besonders günstiges Verhältnis von Aufwand und Ertrag. Entsprechend stehen Umfragedaten zur Lebenszufriedenheit für eine Vielzahl von Ländern und Zeiträumen zur Verfügung. Für viele Zwecke haben sich Befragungen zur Lebenszufriedenheit als gute Methode erwiesen. Bislang handelt es sich um die beste empirische Annäherung an das Konzept der individuellen Wohlfahrt, das in der Ökonomik verwendet wird. Die Zukunft wird zeigen, in welchem Masse und für welche spezifischen Themenbereiche die Glücksforscher sich der unterschiedlichen Erhebungsansätze bedienen werden.

In der Wissenschaft besteht heute ein breiter Konsens, dass sich Erfahrungsnutzen und individuelle Wohlfahrt mit einiger Genauigkeit messen lassen. Die abgefragten Messungen des Wohlbefindens korrelieren hoch mit Verhaltensweisen und Merkmalen, die im Allgemeinen mit Glück und Zufriedenheit assoziiert

4 Diener, E. u.a.: Subjective Well-Being: Three Decades of Progress, in: Psychological Bulletin, 125/2 (1999), 276-302; Kahneman, D./Diener, E./Schwarz, N. (Hg.): Well-Being: The Foundations of Hedonic Psychology, New York, 1999; Kahneman, D./Krueger, A. B.: Developments in the Measurement of Subjective Well-Being, in: Journal of Economic Perspectives, 20/1 (2006), 3-24.

5 Eine nicht auf Befragungen aufbauende Methode der Erfassung des subjektiven Wohlbefindens ist das Brain Imaging. Dabei wird die Magnetresonanztomografie (fMRT) verwendet, um die Entsprechung positiver oder negativer Affekte im Gehirn zu lokalisieren. 
werden. Untersuchungen zur Reliabilität haben gezeigt, dass das geäußerte subjektive Wohlbefinden stabil ist, gleichzeitig jedoch auf sich verändernden Lebensumständen reagiert. Konsistenzüberprüfungen zeigen, dass das geäußerte Lebenszufriedenheitsniveau mit objektiv beobachtbaren Indikatoren von Glück korreliert: Glückliche Menschen lächeln während sozialer Interaktionen häufiger, werden von Freunden, Familienangehörigen und Ehepartnern als glücklich eingestuft, bringen häufiger positive Gefühle zum Ausdruck, sind optimistischer, geselliger und extrovertierter und schlafen besser. ${ }^{6}$

Glücksmessungen lassen sich selbstverständlich immer weiter verbessern. Es gilt beispielsweise weiter zu untersuchen, inwieweit ein Unterschied zwischen den hedonistischen Erfahrungen der Menschen und ihren expliziten reflektierenden Beurteilungen dieser Erfahrungen im geäußerten subjektiven Wohlbefinden besteht. ${ }^{7}$ Mitunter sind Menschen vollauf mit herausfordernden Aktivitäten beschäftigt und erfahren dabei große Freude (oder gar ein ,flow"-Erlebnis, einen Tätigkeitsrausch). Allein schon aufgrund der Eigenart dieser Situation werden Menschen in einem solchen Zustand ihr Wohlbefinden nicht bewerten oder äußern. Zukünftige Forschungen über den Zusammenhang zwischen geäußertem subjektiven Wohlbefinden und physiologischen Messungen des Wohlbefindens werden weitere Aufschlüsse erlauben. ${ }^{8}$

$\mathrm{Zu}$ bedenken ist auch, inwiefern Messungen, die sich auf positive und negative Affekte konzentrieren, den unterschiedlichen Konzepten des Glücks entsprechen, wie sie in der Literatur zum guten Leben vorgeschlagen werden. ${ }^{9}$ Hedonistisches Wohlbefinden ist nicht unbedingt das Gleiche wie Glück oder eine umfassende Zufriedenheit mit dem Leben. Gemäß der eudämonistischen Glücksauffassung sollten die Menschen entsprechend ihrem wahren Ich (daimon) leben. Dafür entscheidende Faktoren sind Selbstbestimmung, Autonomie, Kompetenz und Verbundenheit.

6 Frey, B.S./Stutzer, A.: What Can Economists Learn from Happiness Research?, a.a. O.; Frey, B. S.: Happiness: A Revolution in Economics, a.a.O.

7 Schooler, J.W./Ariely, D./Loewenstein, G.: The Pursuit and Assessment of Happiness can be SelfDefeating, in: Brocas, I./Carrillo, J.D. (Hg.). The Psychology of Economic Decisions. Rationality and Well-Being, Bd. 1, Oxford, 2003, 41-70.

8 Urry, H.L. u.a.: Making a Life Worth Living: Neural Correlates of Well-Being, in: Psychological Science, 15/6 (2004), 367-372.

9 Ryan, R.M./Deci, E. L.: To be Happy or to be Self-Fulfilled: A Review of Research on Hedonic and Eudaimonic Well-Being, in: Annual Review of Psychology, 52 (2001), 141-166. 
Auch wenn die Messungen des Glücks sicherlich kritisierbar sind, sollten sie mit den heute üblichen Wohlfahrtsmessungen verglichen werden, insbesondere mit dem Sozialprodukt, das keine vernünftige Erfassung des individuellen menschlichen Wohlbefindens erlaubt. ${ }^{10}$

\section{Anwendungsmöglichkeiten der ökonomischen Analyse des Glücks}

Vier Anwendungsbereiche der ökonomischen Glücksforschung sind von besonderem Interesse.

\section{Glück als Wohlfahrtsmaß}

Mit dem subjektiven Wohlbefinden steht ein neues umfassendes Wohlfahrtsmaß zur Verfügung. Es zeigt, wie glücklich verschiedene Bevölkerungsgruppen sind (wie beispielsweise Reiche gegenüber Armen oder Alte gegenüber Jungen), wie zufrieden mit ihrem Leben Menschen in unterschiedlichen Ländern sind und wie sich das Glück der Leute über die Zeit verändert. Damit lassen sich interessant Fragen beantworten: Ist es wahr - wie die Kulturpessimisten argumentieren dass die Menschen immer unglücklicher werden? Oder ist die Vorstellung jener Ökonomen richtig, die davon ausgehen, dass das starke Einkommenswachstum der letzten Dekaden die Menschen glücklicher gemacht hat?

\section{Bestimmungsgründe des Glücks}

Es lassen sich mindestens fünf unterschiedliche Gruppen von Determinanten des menschlichen Wohlbefindens unterscheiden. Um den Zusammenhang zwischen den einzelnen Faktoren und dem geäußerten subjektiven Wohlbefinden zu quantifizieren, werden fortgeschrittene ökonometrische Methoden - insbesondere multiple ordered-probit-Schätzungen - verwendet. Diese statistischen Analysen werden für jeweils genau definierte Gruppen von Personen durchgeführt. Alle nachfolgenden Aussagen beziehen sich auf eine isolierte Variation des betrachteten Faktors, d.h. alle anderen Einflüsse werden konstant gehalten (sog. ceterisparibus-Vorgehen). Wenn also etwa der Einfluss des Einkommens auf die Le-

10 New Economics Foundation: National Accounts of Well-being: Bringing Real Wealth into the Balance Sheet, London, 2009; Stiglitz, J.E./Sen, A./Fitoussi, J.-P.: Report by the Commission on the Measurement of Economic Performance and Social Progress, 2009, http://www.stiglitz-sen-fitoussi.fr/documents /rapport_anglais.pdf. 
benszufriedenheit analysiert wird, werden nur darüber Aussagen gemacht, wie das Einkommen mit dem subjektiven Wohlbefinden statistisch zusammenhängt, nicht aber darüber, dass Menschen mit höherem Einkommen oft gesünder sind, was auch wiederum ihre Lebenszufriedenheit erhöht (letzteres wird beim isolierten Einfluss der Gesundheit auf das Glück erfasst).

Die ökonomische Glücksforschung untersucht nicht nur wirtschaftliche Determinanten, sondern bezieht viele verschiedene Einflussfaktoren mit ein. In der Tat konzentrieren sich die drei ersten Gruppen auf außerhalb der Wirtschaft liegende Einflüsse.

\section{a) Genetische Einflüsse}

Menschen sind ihrer Natur nach unterschiedlich glücklich. Jeder wird schon beobachtet haben, dass einige Leute sonnige Gemüter haben und trotz zuweilen schwieriger Umstände mit ihrem Leben im Großen und Ganzen zufrieden sind. Andere Personen nehmen alles schwer, sind pessimistisch und entsprechend weniger glücklich. Diese Unterschiede werden in der ökonomischen Glücksforschung empirisch festgestellt, nicht aber theoretisch interpretiert, denn das ist eindeutig die Angelegenheit anderer Wissenschaften.

Personen weichen von dieser Grundlinie des Glücks (set level) vorübergehend oder auch langfristig ab, wenn sich in ihrem Leben entsprechende Ereignisse einstellen. Wer zum Beispiel einen schweren Unfall erleidet und querschnittgelähmt wird, erleidet eine Phase großen Unglücks. Die Betroffenen sind jedoch (im Durchschnitt) dazu fähig, sich zu erholen, so dass ihr subjektives Glücksniveau nach einiger Zeit wieder deutlich ansteigt und sich der Grundlinie annähert. Umgekehrt erfahren Personen, die unerwartet beim Lotto eine große Geldsumme gewonnen haben, einen positiven Glücksschub, der jedoch nach einiger Zeit wieder deutlich abnimmt. Nach einiger Zeit sind die „Glückspilze“ kaum noch glücklicher als diejenigen, die nicht gewonnen haben. Bemerkenswert ist jedoch, dass diese Tendenz zur Rückkehr zur Grundlinie des Glücks je nach Ereignis unterschiedlich lange dauert und unterschiedlich vollständig geschieht. Zum Beispiel gewöhnen sich arbeitslose Männer - entgegen geläufiger Meinung kaum an ihre Situation (Frauen gelingt dies eher). Hingegen sind Menschen fähig, sich nach Ereignissen wie dem Tod des Partners oder einer Scheidung nach Ablauf einiger Zeit wieder beinahe ganz an die Grundlinie ihres Wohlbefindens anzunähern. 


\section{b) Sozio-demographische Einflüsse}

Verheiratete Personen sind mit ihrem Leben zufriedener als solche, die unverheiratet mit einem Partner leben, und diese wiederum als solche ohne Partner. Sorgfältige Untersuchungen (unter Verwendung von Paneldaten, etwa dem deutschen Sozio-ökonomischen Panel) können zeigen, dass die ursächliche Beziehungen in beide Richtungen wirken ${ }^{11}$ : Glückliche Personen finden leichter einen Partner als unglückliche, ebenso sind Verheiratete glücklicher, wohl weil sie das Gefühl der Sicherheit und der Nähe hoch schätzen. Obige Aussagen zu den Gewöhnungseffekten gelten auch für die Heirat: Zum Zeitpunkt der Eheschließung hin steigt das Glücksgefühl markant an, fällt danach aber wieder deutlich. Allerdings trifft dies nur für die heute fast allein vorherrschende „romantische Heirat“ zu. In Ländern mit dominant arrangierten Ehen ist keine Glückssteigerung zur Heirat hin festzustellen - dafür aber auch nicht der darauf folgende Rückgang.

All diese Aussagen gelten natürlich nur im Durchschnitt. So kann es sehr wohl Menschen geben, die infolge ihrer Heirat ein ganzes Leben lang glücklicher sind. Dafür gibt es auch jene, die von ihrer Heirat schwer enttäuscht sind und sich deshalb unglücklich fühlen.

Einige Studien stellen eine etwas höhere Lebenszufriedenheit von Frauen gegenüber Männern fest, wobei allerdings der Unterschied klein (und statistisch nicht signifikant) ist. Zumindest lässt sich sagen, dass sich Frauen nicht grundsätzlich weniger glücklich fühlen als Männer.

\section{c) Kulturelle Einflüsse}

Zwischen Ländern zeigen sich erhebliche Unterschiede im Niveau der geäußerten Lebenszufriedenheit, unabhängig von anderen Einflüssen. So herrscht in den Vereinigten Staaten geradezu ein sozialer Zwang, sich als ,, happy“ zu bezeichnen; Miesepeter werden dort wenig geschätzt. Umgekehrt gilt es in Frankreich fast als peinlich, sich als , heureux “ zu bezeichnen, denn das weist auf fehlende menschliche Tiefe hin (die meisten französischen Philosophen sind dafür ein treffendes Beispiel). De Gaulle wird der Ausspruch zugeschrieben: „Nur Idioten sind glücklich“. Diese Einstellung gilt auch für die Italiener und trifft eingeschränkt auch für die Deutschen zu (hierzulande ist ja das Gefühl des Weltschmerzes nicht unbekannt).

11 Stutzer, A./Frey, B.S.: Does marriage make people happy, or do happy people get married?, in: Journal of Socio-Economics, 35/2 (2006), 326-347. 
Religiöse Personen erweisen sich als glücklicher als agnostische Personen, die keiner Religionsgemeinschaft angehören. Wer an ein höheres Wesen glaubt und ihm vertraut, kommt besser mit den Widrigkeiten des Lebens zurecht. Einem Schicksalsschlag kann ein höherer Sinn zugeordnet werden, was das seelische Gleichgewicht stärkt. Gleichzeitig führt die Ausübung des Glaubens in einer Religionsgemeinschaft zu intensiveren sozialen Kontakten und wirkt einer Isolation entgegen. Eine der wichtigsten Ergebnisse der Glücksforschung ist in der Tat, dass soziale Kontakte innerhalb der Familie und im Freundes- und Bekanntenkreis wesentlich zur Lebenszufriedenheit beitragen.

\section{d) Wirtschaftliche Einflüsse}

Die ökonomische Glücksforschung konzentriert sich auf den Einfluss wirtschaftlicher Faktoren auf die Lebenszufriedenheit. Ein höheres Einkommen ist im unteren Teil der Einkommensverteilung mit deutlich höherem subjektivem Wohlbefinden verbunden. Ob reich oder sehr reich spielt für die geäußerte Lebenszufriedenheit jedoch kaum eine Rolle. Interessanterweise gibt es auch beim Einkommen starke Gewöhnungseffekte. Während eine Einkommenssteigerung kurzfristig das Wohlbefinden stark erhöht, gehen innerhalb von ein, zwei Jahren zwischen zwei Drittel und drei Viertel des Effektes wieder verloren. Von großer Bedeutung für das subjektive Wohlbefinden ist der Vergleich des eigenen Einkommens mit dem von Personen in der Referenzgruppe. Wenn dessen Angehörige höhere Einkommenssteigerungen als man selbst erleben, kann die eigene Lebenszufriedenheit sogar zurückgehen, obwohl das eigene Einkommen steigt.

Wichtige Einsichten hat die Glücksforschung hinsichtlich der Rolle der Arbeit gebracht. Wer arbeitslos wird - so zeigen alle Untersuchungen - ist wesentlich unzufriedener als ein Beschäftigter. Dieses Ergebnis ist deshalb überraschend, weil - wie oben erklärt - das Einkommen statistisch konstant gehalten wird und nur die isolierte Wirkung der Arbeitslosigkeit an sich betrachtet wird. Der Grund für den stark negativen Effekt liegt im psychologischen Stress, der aus dem Ausschluss vom Rest der (arbeitenden) Gesellschaft und der beeinträchtigten Selbsteinschätzung herrührt. In der Tat sind Arbeitslose, die in einer Region mit vielen anderen Arbeitslosen wohnen, weniger unglücklich als solche, die von Menschen mit Arbeit umgeben sind.

Nimmt die Arbeitslosenquote in einem Land zu, leiden nicht nur die Arbeitslosen selbst, sondern auch der Rest der Bevölkerung. Sie sind weniger zufrieden, weil 
mit einer höheren Arbeitslosigkeit eine generell höhere wirtschaftliche Unsicherheit einhergeht, insbesondere eine stärkere Sorge um die eigene Stelle. ${ }^{12}$

Auch die Zufriedenheit mit einer bestimmten Arbeit wurde ausführlich untersucht. Wer eine möglichst autonome Arbeit mit einem großen Grad an Selbstbestimmung durchführen kann, ist zufriedener als wenn der Arbeitgeber alles bestimmt und vorschreibt. Aus diesem Grund äußern Selbständige eine höhere Arbeitszufriedenheit als die abhängig Beschäftigten.

Bemerkenswert ist auch der Einfluss der Einkommensungleichheit, weil sich der statistische Zusammenhang mit dem subjektiven Wohlbefinden zwischen den Vereinigten Staaten und Europa grundsätzlich unterscheidet. Amerikaner in Bundesstaaten mit stärkerer Einkommensungleichheit geben weder an glücklicher noch unglücklicher zu sein. Eine Erklärung dafür wäre, dass sie den Reichtum der Individuen im oberen Teil der Einkommensverteilung als Indiz dafür nehmen, dass sich harte Arbeit lohnt und zu höherem Einkommen führt. Sie glauben, dass sie selbst davon profitieren können. In Europa hingegen sind die Leute eher davon überzeugt, dass die Aufwärtsmobilität gering ist und sie deshalb an der höheren Einkommensdisparität nicht teilhaben werden. Vorliegende Forschungen zeigen allerdings, dass sich die Aufwärtsmobilität in Europa nicht wesentlich von der in den USA unterscheidet. Dies ist ein Indiz dafür, dass subjektive Einschätzungen und Vorstellungen und nicht die tatsächlichen Umstände von entscheidender Bedeutung für die Wirkung auf die Lebenszufriedenheit sind.

\section{e) Politische Institutionen}

Die ökonomische Analyse des Glücks ermöglicht auch Beiträge zur Politischen Ökonomie. Bisher hat sich die Forschung eingehend mit der Frage beschäftigt, ob und wie sich institutionelle Rahmenbedingungen und Politiksysteme auf ökonomisch relevante Größen auswirken. Zum Beispiel wurde untersucht, ob die Wirtschaftsleistung in einer demokratischen Gesellschaft höher ist als in einer autoritären Gesellschaft. Die Glücksforschung kann entscheidend zu dieser Literatur beitragen. Es wurde zum Beispiel untersucht, welcher Zusammenhang zwischen Glück und Demokratie besteht. Diese Frage ist nicht einfach zu beantworten, weil zwei gegenläufige Kausalbeziehungen zu berücksichtigen sind. Auf der einen Seite können demokratische Verhältnisse die Menschen zufriedener

12 Lüchinger, S./Meier, S./Stutzer, A.: Why Does Unemployment Hurt the Employed? Evidence from the Life Satisfaction Gap between the Public and the Private Sector, in: Journal of Human Resources, 45 (2010), i. E. 
machen; auf der andern Seite ist es auch denkbar, dass sich Gesellschaften mit mehrheitlich zufriedenen Menschen für mehr Demokratie entscheiden, respektive zufriedene Menschen die Demokratie stabilisieren.

Für den positiven Einfluss der Demokratie auf das Glück sind zwei Gründe maßgeblich. Erstens entsprechen die politischen Entscheidungen in Demokratien eher den Wünschen (Präferenzen) der Bürger. Die Politiker sind wegen der Wiederwahlrestriktion gezwungen, diejenigen staatlichen Güter und Dienstleistungen anzubieten, und diejenigen Regulierungen durchzuführen, die von ihren Wählern nachgefragt werden. Zweitens schätzen die Individuen die Möglichkeit politischer Teilnahme an und für sich, auch ganz unabhängig vom Ergebnis. Diese Abhängigkeit des Nutzens von der Art und Weise, wie ein Ergebnis zustande kommt, wird als „Prozessnutzen“"13 bezeichnet - ein Zusammenhang der in der Rechtswissenschaft wohlbekannt ist, jedoch nun auch empirisch nachgewiesen ist. Der gefundene Zusammenhang zwischen Demokratie und Lebenszufriedenheit entspricht dem psychologischen Grundbedürfnis nach Selbstbestimmung. Die Bedeutung dieser beiden Faktoren für die Lebenszufriedenheit konnte anhand der unterschiedlichen Partizipationsmöglichkeiten zwischen den 26 Schweizer Kantonen gezeigt werden: In denjenigen Kantonen, in denen sich die Bürger mit geringen Hürden mittels Initiativen und Referenden an der Entscheidungen beteiligen können, sind sie zufriedener als in denjenigen Kantonen, in denen diese Möglichkeit stärker eingeschränkt ist (zum Beispiel fiskalische Aspekte weitgehend ausgeschlossen sind). Interessanterweise ist der statistische Zusammenhang stärker für Schweizer als für Ausländer. Ausländer profitieren zwar in (etwa) gleicher Weise von den besseren politischen Entscheidungen, sind hingegen von der politischen Entscheidungsbildung ausgeschlossen. Zur Klarheit sei noch einmal betont, dass bei diesen Untersuchungen die übrigen Einflüsse auf die Lebenszufriedenheit berücksichtigt werden. Die beobachteten Unterschiede zwischen den Kantonen mit unterschiedlichem Grad an politischen Mitwirkungsmöglichkeiten gehen deshalb nicht auf andere Faktoren (wie etwa unterschiedliches Einkommen oder unterschiedliche Arbeitslosigkeit) zurück.

Die Lebenszufriedenheit der Bürger ist auch umso höher, je mehr Entscheidungen auf der lokalen Ebene getroffen werden. Dies lässt sich damit erklären, dass sie das Gefühl haben, dass ihre Anliegen ernster genommen werden als wenn die

13 Frey, B.S./Benz, M./Stutzer, A.: Introducing Procedural Utility: Not Only What, but Also How Matters, in: Journal of Institutional and Theoretical Economics, 160/3 (2004), 377-401. 
Entscheidungen weit entfernt auf einer zentralen politischen Ebene gefällt werden.

\section{Psychologische Prozesse und menschliches Wohlbefinden}

Angaben zur subjektiven Beurteilung der Lebensqualität helfen die psychologischen Mechanismen zu analysieren, die das menschliche Wohlbefinden bestimmen. Das subjektive Wohlbefinden setzt sich aus den zwei grundlegenden Aspekten Kognition und Affekt zusammen. Affekt ist die Bezeichnung für Stimmungen und Emotionen. Affekte repräsentieren die unmittelbare Evaluation von Ereignissen, die im Leben der Individuen geschehen. Die kognitive Komponente bezieht sich auf die rationalen oder intellektuellen Aspekte des subjektiven Wohlbefindens. Sie wird üblicherweise anhand von Maßen zur Zufriedenheit beurteilt. Angenehme Affekte, unangenehme Affekte und Lebenszufriedenheit erweisen sich als trennbare Konstrukte. Der kognitive Aspekt schließt die beiden wichtigen Komponenten Vergleiche und Urteilsbildung ein. Das subjektive Wohlbefinden ist nicht vorgegeben und unveränderlich, sondern wird in der befragten Person konstruiert und hängt stark von der sozialen Umwelt ab.

Zum Verständnis der menschlichen Wohlfahrt sind insbesondere drei psychologische Prozesse wichtig:

- Adaptation: Individuen gewöhnen sich an neue Umstände und ihr subjektives Wohlbefinden passt sich entsprechend an. Die hedonische Adaptation führt dazu, dass Individuen weniger auf wiederholte oder kontinuierliche Stimuli ansprechen. ${ }^{14}$

- Anspruchsniveau: Menschen beurteilen ihre Situation selten absolut, sondern meist in Bezug auf ihr Anspruchs- oder Aspirationsniveau, das stark durch ihre Erwartungen beeinflusst wird. Je besser sich ihre Lebenssituation relativ zum Anspruchsniveau präsentiert, desto höher ist ihre Zufriedenheit mit dem Leben. ${ }^{15}$

- Soziale Vergleiche: Referenzmaßstäbe für subjektive Urteile bilden sich wesentlich durch Vergleiche mit Personen in der eigenen Umgebung, zum Beispiel den Arbeitskollegen.

14 Frederick, S./Loewenstein, G.: Hedonic Adaptation, in: Kahneman, D./Diener, E./Schwarz, N. (Hg.): Well-Being: The Foundations of Hedonic Psychology, New York, 1999, 302-329.

15 Easterlin, R.A.: Income and Happiness: Towards a Unified Theory, in: Economic Journal, 111/473 (2001), 465-484. 


\section{Auswirkungen auf das Verhalten.}

Die Maße für das subjektive Wohlbefinden erlauben es, dessen Auswirkungen auf das Verhalten zu erfassen. ${ }^{16}$ Ob Menschen glücklich oder unglücklich sind, hat einen großen Einfluss darauf, wie sie in verschiedenen Bereichen leben und handeln. So sind glückliche Menschen beispielsweise erfolgreicher auf dem Arbeitsmarkt. Glückliche Menschen sind auch kooperativer; sie sind eher bereit, anderen zu helfen und dabei Risiken auf sich zu nehmen. Wie diese Beispiele zeigen, ist es in vielen Fällen schwierig, die Richtung der Kausalität zu bestimmen. So sind Menschen auch glücklicher, weil sie beruflich erfolgreicher sind als andere.

\section{Bewertung öffentlicher Güter}

\section{Der Ansatz der Lebenszufriedenheit}

Öffentliche Güter sind dadurch definiert, dass niemand von deren Konsum ausgeschlossen werden kann und der Konsum nicht rivalisierend ist. Aus diesem Grund besteht kein Markt, auf dem sich die Zahlungsbereitschaft der Konsumenten spiegelt. Um Präferenzen für öffentliche Güter dennoch messen zu können, wurde eine Vielzahl unterschiedlicher Verfahren entwickelt. ${ }^{17}$ Mit dem geäußerten subjektiven Wohlbefinden als Messgröße für die individuelle Wohlfahrt lassen sich nunmehr auch öffentliche Güter unmittelbar bewerten. Dieses Verfahren ist als Lebenszufriedenheitsansatz (life satisfaction approach, $\mathrm{LSA}^{18}$ ) bekannt.

Mit Hilfe des LSA lassen sich eine Vielzahl verschiedener öffentlicher Güter und Übel sowie negativer und positiver Externalitäten bewerten. Bislang wurde dieser Ansatz hauptsächlich dazu verwendet, um Externalitäten im Umweltbereich zu bewerten. Van Praag und Baarsma untersuchen, welche Auswirkung die Lärmbelästigung im Bereich des Amsterdamer Flughafens hat. ${ }^{19}$ Lüchinger

16 Lyubomirsky, S./King, L./Diener, E.: The Benefits of Frequent Positive Affect: Does Happiness Lead to Success?, in: Psychological Bulletin, 131/66 (2005), 803-855.

17 Freeman, A. M. III.: The Measurement of Environmental and Resource Values: Theory and Methods, 2. Aufl., Washington, DC, 2003.

18 Frey, B.S./Lüchinger, S./Stutzer, A.: The Life Satisfaction Approach to Environmental Valuation, in: Annual Review of Resource Economics, 2010, i. E.

19 Van Praag, B.M. S./Baarsma, B.E.: Using Happiness Surveys to Value Intangibles: The Case of Airport Noise, in Economic Journal, 115/500 (2005), 224-246. 
kommt in einer Untersuchung für Deutschland zu dem Ergebnis, dass sich die Schwefeldioxidbelastung negativ auf die durchschnittliche Lebenszufriedenheit auswirkt und signifikante Wohlfahrtskosten hat. ${ }^{20}$

Der Lebenszufriedenheitsansatz hat mehrere Vorteile gegenüber den Standardmethoden, die man gegenwärtig verwendet, also etwa gegenüber dem hedonischen Preisansatz oder kontingenten Bewertungsmethoden.

\section{Eine Anwendung auf die Kosten des Terrorismus}

Das Wohlbefinden der Bürger wird durch Terrorismus sicherlich negativ beeinflusst. Menschen, die in einem vom Terrorismus betroffenen Land leben, sind weniger glücklich als diejenigen, die in geordneten politischen Verhältnissen leben. Ein Beispiel dafür ist die Dominikanische Republik im Jahr 1962, als nach dem Mord an Präsident Trujillo die politische Situation aufgewühlt und das Land in ein politisches Chaos zu geraten drohte. Die in diesem Land gemessene Einschätzung des Lebens lag (auf einer Skala von 0 für das schlimmstmögliche Leben bis 10 für das bestmögliche Leben) bei 1,6 - der niedrigste je verzeichnete Wert. In politisch stabilen Demokratien hingegen wie etwa in der Schweiz, in Norwegen oder in Dänemark gibt die Bevölkerung eine hohe Lebenszufriedenheit an. So lagen die entsprechenden Werte in den 1990er Jahren bei 8,2 (Dänemark), 8,0 (Schweiz) und 7,7 (Norwegen). Glück und politische Stabilität scheinen somit eng zusammenzuhängen. ${ }^{21}$

Die Kausalität verläuft jedoch möglicherweise in beiderlei Richtungen: Politische Unruhen machen die Menschen unzufrieden, gleichzeitig demonstrieren, streiken oder verüben unzufriedene Menschen Terroranschläge, was zu politischer Instabilität führt. Allerdings werden die meisten Staatsstreiche und Revolutionen von konkurrierenden politischen Clans, Parteien oder dem Militär angezettelt.

Mit Hilfe des LSA lassen sich die Kosten des Terrorismus systematisch bewerten. Auf der Grundlage von Paneldaten ist es möglich, die Lebenszufriedenheit der Bevölkerung in vom Terror betroffenen Regionen und Städten über die Zeit mit Teilen des Landes zu vergleichen, in denen der Terrorismus geringer ist oder

20 Lüchinger, S.: Valuing Air Quality Using the Life Satisfaction Approach, in: Economic Journal, 119/ 536 (2009), 482-515.

21 Wir sind uns natürlich bewusst, dass auch andere Faktoren für den festgestellten Unterschied verantwortlich sind. 
ganz fehlt. Dieser Ansatz wird hier am Fall Frankreich exemplifiziert, wurde jedoch auch auf andere Länder angewendet. ${ }^{22}$

Die Daten zur Lebenszufriedenheit stammen aus der Eurobarometer-Umfrage (1970-1999); die ermittelte Variable ergibt sich aus der Antwort auf die Frage: „Sind Sie insgesamt gesehen mit dem Leben, das Sie führen, sehr zufrieden [4], ziemlich zufrieden [3], nicht besonders zufrieden [2] oder überhaupt nicht zufrieden [1]?“ Als Indikator für Häufigkeit und Intensität terroristischer Aktivitäten dient die Zahl terroristischer Anschläge. Die beiden vom Terrorismus stark betroffenen Regionen Île-de-France (einschließlich Paris) und Provence-AlpesCôte-d'Azur (zu der in der Eurobarometer-Umfrage auch Korsika gehört) werden für die Jahre 1973-1998 mit dem Rest Frankreichs verglichen. Mit diesen Datensätzen wird die Lebenszufriedenheit der Menschen, die zu einer bestimmten Zeit in einer bestimmten Region lebten, durch das je nach Region und über die Zeit unterschiedliche Terrorniveau, das Haushaltseinkommen der Person, andere persönliche und soziodemografische Merkmale sowie summarische regionenund zeitspezifische Effekte erklärt.

Aus den ökonometrischen Regressionsschätzungen ergibt sich, dass die Zahl der Terroranschläge einen statistisch signifikanten negativen Effekt auf die geäußerte Lebenszufriedenheit hat. Bei 15 Terroranschlägen (also in etwa die durchschnittliche Zahl der Attacken in Paris während des Untersuchungszeitraums) folgt eine durchschnittliche Verringerung der Lebenszufriedenheit um 0,04 auf der VierPunkte-Skala. Die Lebenszufriedenheit sinkt damit um rund ein Fünftel des Werts, den eine Person im Durchschnitt erleidet, wenn sie arbeitslos wird.

Mit Hilfe der geschätzten partiellen Korrelation für das Einkommen lässt sich die hypothetische Zahlungsbereitschaft für eine Veränderung des Ausmaßes an terroristischer Aktivität messen. Zu Vergleichszwecken wird der Unterschied im Ausmaß an Terrorismus zwischen der Region Ille-de-France (Paris) und im Rest Frankreichs (außer Provence-Alpes-Côte-d'Azur) herangezogen. Entsprechend wäre ein Bewohner von Paris (mit durchschnittlichem Haushaltseinkommen) bereit, rund 4 bis $8 \%$ seines Einkommens zu bezahlen, damit der Terror auf ein Niveau reduziert wird, wie es in den friedlicheren Teilen des Landes herrscht. 


\section{Politik als Glücksmaximierung?}

Auf Grundlage der Ergebnisse der Glücksforschung haben verschiedene Autoren gefolgert, dass die Regierungen das Glück ihrer Einwohner maximieren sollen. ${ }^{23}$ Die Politiker sollen demnach die oben diskutierten Ergebnisse der Glücksforschung direkt umsetzen. Damit würde der Traum der "Theorie quantitativer Wirtschaftspolitik“ im Anschluss an den Nobelpreisträger Tinbergen (1969) und Theil in die Tat umgesetzt. Diese Wissenschaftler postulieren, dass der Staat eine gesellschaftliche Wohlfahrtsfunktion maximieren soll. Bisher war es jedoch nicht möglich, diese gesellschaftliche Wohlfahrtsfunktion konkret zu spezifizieren. Die Glücksforschung gibt nun die Möglichkeiten dazu.

Die Moderne Politische Ökonomie hat aber seit langem grundsätzliche und überzeugende Argumente gegen diesen Ansatz vorgebracht. Insbesondere die „ökonomische Theorie der Verfassung ${ }^{\text {‘24 }}$ hat gezeigt, dass der Versuch einer Maximierung der gesellschaftlichen Wohlfahrt der Vorstellung eines ,wohlwollenden Diktators" entspricht, der oder die von oben bestimmt, was für die Menschen zu gelten hat. Den Politikern, die diese Maßnahmen durchzuführen haben, wird damit unterstellt, dass sie einzig und allein das Interesse ihrer Einwohner im Auge haben. Eine solche Annahme ist naiv und widerspricht auch der ökonomischen Grundannahme, dass Individuen vor allem ihren eigenen Nutzen verfolgen. Weit sinnvoller und empirisch gut begründet ist die Annahme, dass Politiker vor allem am Machterhalt interessiert sind. In demokratischen Gesellschaften müssen Politiker Wahlen gewinnen, damit sie an der Regierung blieben können. Dazu unternehmen sie alle ihnen zur Verfügung stehenden Maßnahmen. Insbesondere werden unpopuläre Maßnahmen in die Zukunft verschoben. Sie sind durchaus bereit, ihre Popularität mit kurzfristig wirkenden Wahlgeschenken zu erkaufen. Derartiges politisches Handeln widerspricht dem langfristigen Interesse der Bevölkerung, wird aber von dieser nur unzureichend bemerkt und deshalb bei den Wahlen nicht bestraft. Nur wenn die Personen an der Regierung von ihrer Wiederwahl hinreichend überzeugt sind, führen sie inhaltliche Programme durch, die ihren eigenen Überzeugungen entsprechen. Dazu kann gewiss auch gehören, dass sie das Glück der Menschen erhöhen möchten. Allerdings geht es

23 Vgl. insbesondere Layard, R.: Happiness: Lessons from a New Science, New York, 2005.

24 Buchanan, J.M.: Social Choice, Democracy, and Free Markets, in: Journal of Political Economy, 62/2 (1954), 114-123; Buchanan, J.M./Tullock, G.: The Calculus of Consent. Logical Foundations of Constitutional Democracy, Ann Arbor, 1962; Frey, B. S.: Moderne Politische Ökonomie, München, 1977. 
dabei um deren persönliche Interpretation dessen, was die Ergebnisse der Glücksforschung darlegen.

Verfolgt eine Regierung eine Politik der Glücksmaximierung, ergibt sich ein weiteres schwerwiegendes Problem. Die Ergebnisse der Glücksforschung bauen auf sorgfältigen Umfragen auf, bei denen die befragten Personen unbeeinflusst aussagen, wie zufrieden sie sind. Wenn jedoch die Befragten wissen, dass ihre Antworten für politische Zwecke verwendet werden, werden sie ihr Verhalten ändern und die Auswirkungen auf die Politik berücksichtigen. So hat zum Beispiel eine Person, die den Konservativen zuneigt, Anlass, sich als besonders glücklich zu bezeichnen, wenn eine konservative Partei an der Regierung ist. Umgekehrt tendiert sie dazu, eine linke Regierung zu „bestrafen“, indem sie sich als weniger glücklich ausgibt, als sie in Wirklichkeit ist. Dieses Verhalten untergräbt die Aussagekraft der Umfrageforschung und damit weitgehend auch der empirischen Glücksforschung.

Die Glücksforschung hat durchaus politische Bedeutung, wenn auch nicht im Sinne einer kruden Maximierung des Glücks der Bevölkerung. Es ist nützlich, zwei Ebenen der Politik zu unterscheiden:

- Im laufenden politischen Prozess, in dem die Verfassung als vorgegeben betrachtet wird, können Politiker an der Regierung wie auch in der Opposition Anregungen aus den Ergebnissen der Glücksforschung ziehen. Sie werden dies insbesondere tun, wenn sie sich daraus einen Vorteil im Wettbewerb um Wählerstimmen versprechen. Insbesondere können sie zur Kenntnis nehmen, dass Arbeitslosigkeit zu erheblichen Einbußen an Glück führt. Dies gilt natürlich insbesondere für die Personen, die ihre Stelle verlieren. Eine zunehmende Arbeitslosigkeit in einer Gesellschaft vermindert jedoch auch das Glücksniveau der Beschäftigten. Sie fürchten, in der $\mathrm{Zu}$ kunft selbst arbeitslos zu werden oder dass soziale Konflikte entstehen, möglicherweise sogar Unruhen und revolutionäre Entwicklungen. Eine Regierung sollte demnach viel Gewicht auf die Verhinderung von Arbeitslosigkeit legen. Im Vergleich dazu sollte sie weniger Gewicht auf das Wirtschaftswachstum legen, denn die Glücksforschung zeigt, dass verschiedene Anpassungsprozesse dazu führen, dass eine Einkommenssteigerung nur zu einer geringen nachhaltigen Steigerung des Glücks führt.

- Auf der konstitutionellen Ebene werden die Institutionen bestimmt, die den politischen und gesellschaftlichen Prozess lenken sollen. Wie oben ausgeführt, hat die Glücksforschung in dieser Hinsicht wichtige Ergebnisse gelie- 
fert. Insbesondere wurde gezeigt, dass die Bürgerinnen und Bürger verbesserte Möglichkeiten zur politischen Mitbestimmung schätzen. Dies bezieht sich vor allem auf die vermehrte Mitsprache bei Sachentscheidungen mittels Volksinitiativen und Referenden. Derartige Möglichkeiten auf zentraler und regionaler Ebene werden in verschiedenen Staaten, darunter auch Deutschland, diskutiert, könnten aber auch eine größere Rolle in der Europäischen Union einnehmen. Darüber hinaus gibt es Evidenz aus der Glücksforschung, dass die Bürgerinnen und Bürger eine föderale Organisation mit starker lokaler Autonomie gegenüber stärker zentralisierten Einheiten vorziehen. Die möglichen institutionellen Veränderungen sollten nicht von der Regierung autoritär verfügt werden, sondern als Vorschläge in den demokratischen Diskursprozess eingehen.

Die Glücksforschung ist durchaus politisch relevant, aber nicht im Sinne einer Glücksmaximierung durch die Regierung. Vielmehr sollte großes Gewicht auf die Schaffung von Institutionen gelegt werden, die es den einzelnen Personen ermöglicht, auf eigene Weise und im Kollektiv möglichst glücklich zu werden. 